\title{
Three-Dimensional Fabry-Perot Imaging Spectroscopy of the Crab Nebula
}

\author{
Stephen S. Lawrence and Gordon M. MacAlpine
}

Dept. of Astronomy, University of Michigan, Ann Arbor, MI 48109, U.S.A.

\author{
Alan Uomoto \\ Dept. of Physics and Astronomy, The Johns Hopkins University, \\ Baltimore, MD 21218, U.S.A.
}

Richard Gelderman, Bruce E. Woodgate, and Larry W. Brown

NASA Goddard Space Flight Center, Greenbelt, MD 20771, U.S.A.

\begin{abstract}
A three-dimensional model of the [O III] $\lambda 5007$ line-emitting gas in the Crab Nebula has been developed from imaging spectroscopy taken with the Goddard Fabry-Perot Imager mounted on the McGrawHill 1.3m telescope of Michigan-Dartmouth-MIT Observatory. Several interesting morphological features revealed in three-dimensional isophotal displays are discussed.
\end{abstract}

\section{Introduction}

The advent of astronomical imaging Fabry-Perot cameras has opened a new window on the study of astronomical objects, allowing us to construct threedimensional data cubes which serve as maps in position-velocity space. With certain reasonable and simple assumptions, the data cube for a young supernova remnant can be translated into a physical model in linear space. This paper presents a brief overview of such a model developed from the [OIII] $\lambda 5007$ emission of the Crab Nebula.

\section{Data Reduction}

A spectral scan of the Crab Nebula was obtained in [O III] $\lambda 5007$ emission using the Goddard Fabry-Perot Imager (GFPI) mounted on the $1.3 \mathrm{~m}$ McGrawHill telescope at Michigan-Dartmouth-MIT Observatory (MDM) in December of 1989. The GFPI itself is described by Gelderman, et al. elsewhere in these proceedings. Individual images within the [O III] scan had exposure times of 300 seconds, bandwidths of $5.3 \AA\left(320 \mathrm{~km} \mathrm{~s}^{-1}\right)$ FWHM, and were spaced $2.1 \AA$ $\left(130 \mathrm{~km} \mathrm{~s}^{-1}\right)$ apart. Initial reductions followed standard procedures for CCD images, employing the data reduction routines within IRAF. Individual images within a scan were normalized to a common level using photometry of stars within the field of view. Stars and the diffuse synchrotron emission were removed 
by appropriately scaling and subtracting frames taken significantly far from nebular line emission. The $(\alpha, \delta, \lambda)$ data cube was translated into a model in linear $(X, Y, Z)$ space using the age (935 yrs) and and an adopted distance $(2,000 \mathrm{pc})$. It was assumed that the filaments have had constant radial velocity throughout the lifetime of the remnant, which is not unreasonable given the relatively young age of the Crab. This minimal assumption also served to keep the model as a linear rescaling of the original $(\alpha, \delta, \lambda)$ data cube. The model was displayed and analyzed using a Stardent $3000 / 750$ Graphics Supercomputer and the Application Visualization System software to render three-dimensional surfaces of constant line intensity. The isosurfaces were color-coded according to radial velocity and could be rotated and viewed from any angle.

\section{Results}

A number of interesting features can be seen in the gaseous structures represented by the data cube (Lawrence et al. 1994). The filament showing the most intense $[\mathrm{O} \mathrm{III}] \lambda 5007$ emission and also the most contiguous extent in velocity space is a formation that extends horizontally in the E-W plane of the sky. This feature corresponds to the "high helium band" detected by Uomoto and MacAlpine (1987) and is shown to be a complete torus, as speculated by MacAlpine et al. (1989). Although complete, this torus is asymmetric in its [O III] emission, with a large fraction of its emission arising from a region slightly west of the center of the nebula and on the blueshifted front side of the torus. The brightest [O III] filaments in the Crab, including the E-W torus, have a conspicuous degree of large-scale organization. Computer-animated rotations of model isophotes are especially effective at drawing attention to this organization, which somewhat resembles the pattern of lines of longitude on a globe. The "polar" axis is marked by the convergence of all of the brighter filaments at two points along the E-W torus near the front and back of the nebula, and is slightly offset from the line of sight to Earth. The "equator" is roughly defined by the zero radial velocity plane. The model confirms that the majority of the line-emitting filaments exist in a thick shell surrounding the central synchrotron cavity, and also reveals a distinct lack of [O III] filaments at the ends of the major (SE-NW) axis of the nebula. When the model is viewed along the major axis from the SE, the brightest filaments fall in projection upon the E-W torus and appear as a ring with a clear line of sight available through the entire nebula.

\section{References}

Gelderman, R., Woodgate, B., and Brown, L. 1994, these proceedings

Lawrence, S.S., MacAlpine, G.M., Uomoto, A., Woodgate, B.E., Brown, L.W., and Oliversen, R.J. 1994, in preparation

MacAlpine, G.M., McGaugh, S.S., Mazzarella, J.M., and Uomoto, A. 1989, ApJ, 342,364

Uomoto, A. and MacAlpine, G.M. 1987, AJ, 93, 1511 